\title{
REGULARITY OF MAPPINGS OF $G$-STRUCTURES OF FROBENIUS TYPE
}

\author{
CHONG KYU HAN
}

(Communicated by Jonathan M. Rosenberg)

\begin{abstract}
A notion of Frobenius type for a $G$-structure is defined. It is shown that a mapping $f$ between $C^{\infty}$ (resp. $C^{\omega}$ ) manifolds with a $G$-structure of the Frobenius type is $C^{\infty}$ (resp. $C^{\omega}$ ) if $f \in C^{k}$, where the integer $k$ depends on the order of the Frobenius type. It is also shown that a $G$-structure of finite order is of the Frobenius type.
\end{abstract}

\section{INTRODUCTION}

Let $G$ be a Lie subgroup of $G l(n ; \mathbf{R})$. A $G$-structure on a $C^{\infty}$ manifold $M$ of dimension $n$ is a $C^{\infty}$ sub-bundle $P$ of the bundle of linear frames over $M$ with structure group $G$. Let $f$ be a $C^{1}$ diffeomorphism of $M$ with a $G$-structure $P$ onto another manifold $\widetilde{M}$ with a $G$-structure $\widetilde{P}$. $f$ is called a $G$-mapping if for any frame field $\left(e_{1}, \ldots, e_{n}\right)$ over $M$ belonging to $P,\left(f_{*} e_{1}, \ldots, f_{*} e_{n}\right)$ is a frame field over $\widetilde{M}$ belonging to $\widetilde{P}$. We are concerning with regularity of $G$-mappings. Locally, the above condition is a system of partial differential equations of order 1 and the question of regularity naturally arises. Our approach is to reduce the regularity problem of $G$-mappings to that of infinitesimal automorphisms of $P$ and then to find conditions on $P$ which imply the regularity of infinitesimal automorphisms. In cases, regularity of infinitesimal automorphisms of a $G$-structure can be deduced by properties of $G$ only. A well-known example is that if the associated Lie algebra of $G$ contains no matrix of rank 1 then an infinitesimal automorphism of a $G$-structure satisfies a system of elliptic linear partial differential equations with $C^{\infty}$ coefficients, and therefore is $C^{\infty}$ (see the proof of Theorem 4.1 of [5]). But in general, we need conditions on a specific $G$-structure $P$ in addition to the conditions on $G$. This paper concerns the cases where regularity of $G$-mappings follows from the Frobenius theorem.

Received by the editors June 18,1986 and, in revised form, July 11, 1988. Presented at the 828th American Mathematical Society Meeting, Logan, October 1986.

1980 Mathematics Subject Classification (1985 Revision). Primary 32F25, 53C10.

Key words and phrases. $G$-structures, infinitesimal automorphisms, Frobenius type, Lie algebras of finite order, CR structures. 
Our viewpoint is purely local, so, for example, a "function" must be understood as a germ of a function at a reference point and in $\S 1$ we work on an open set $\mathscr{O} \subseteq \mathbf{R}^{n}$ instead of a manifold $M$. Often, we have to think of open subsets of $\mathscr{O}$, however, we denote them also by $\mathscr{O}$, for there is no danger of confusion. If the underlying manifolds and $G$-structures on them are real analytic we get a real analytic version of this paper by changing every $C^{\infty}$ to $C^{\omega}$.

\section{MAPPINGS OF $G$-STRUCtURES OF FrobeniUs TYPE}

Let $\mathscr{O}$ be an open subset of $\mathbf{R}^{n}$ and $f=\left(f^{1}, \ldots, f^{l}\right)$ be a system of real valued functions of $\mathscr{O} . f$ is said to satisfy a complete system of order $m$ if every partial derivative of $f^{j}, j=1, \ldots, l$, of order $m$ can be expressed as a $C^{\infty}$ function of the partial derivatives of $\left(f^{1}, \ldots, f^{l}\right)$ of order $<m$ : For each $j=1, \ldots, l$, each multi-index $\alpha$ with $|\alpha|=m$,

$$
D^{\alpha} f^{j}=H_{\alpha}^{j}\left(x, D^{\beta} f:|\beta|<m\right), \quad H_{\alpha}^{j} \in C^{\infty}
$$

where $D^{\beta} f \equiv\left(D^{\beta} f^{1}, \ldots, D^{\beta} f^{l}\right)$.

We will use summation convention in this section: repeated indices denote the summation over $1, \ldots, n$. A complete system for a vector field $X=$ $\xi^{i}\left(\partial / \partial x_{i}\right)$ on $\mathscr{O}$ is a complete system for its components $\left(\xi^{1}, \ldots, \xi^{n}\right)$. If $\mathscr{O}$ is equipped with a $G$-structure $P$ let $\left(e_{1}, \ldots, e_{n}\right)$ be a frame belonging to $P$ and set $L_{X} e_{j}=a_{j}^{i} e_{i}$, where $L$ is the Lie derivative. Then a $C^{1}$ vector field $X$ is an infinitesimal automorphism of $P$ if and only if the matrix $\left[a_{j}^{i}\right]$ belongs to $\mathscr{G}$, the associated Lie algebra of $G$ (cf. [5]). This condition can be expressed as a system of linear partial differential equations of first order: Let $X=u^{i} e_{i}$ and let

Then

$$
\left[e_{i}, e_{j}\right]=b_{i j}^{k} e_{k} .
$$

$$
\begin{aligned}
L_{X} e_{j} & =\left[u^{i} e_{i}, e_{j}\right] \\
& =\left(-e_{j} u^{i}+u^{k} b_{k j}^{i}\right) e_{i},
\end{aligned}
$$

so we have

$$
a_{j}^{i}=-e_{j} u^{i}+u^{k} b_{k j}^{i} \text {. }
$$

Since $\mathscr{G}$ is a linear subspace of $g l(n ; \mathbf{R})$, it is defined by linear equations, namely,

$$
\mathscr{G}=\left\{y_{j}^{i} \in \mathbf{R}^{n^{2}}: c_{i \lambda}^{j} y_{j}^{i}=0, \quad \lambda=1, \ldots, N\right\}
$$

where $N$ is the codimension of $\mathscr{G}$ in $g l(n ; \mathbf{R})$ and $c$ are constants. Thus we get

$$
c_{i \lambda}^{j}\left(-e_{j} u^{i}+u^{k} b_{k j}^{i}\right)=0, \quad \lambda=1, \ldots, N .
$$

To express the above in terms of local coordinates, let $X=\xi^{i}\left(\partial / \partial x_{i}\right)$ and let $\left(\partial / \partial x_{j}\right)=b_{j}^{i} e_{i}$, then $u^{i}=b_{j}^{i} \xi^{j}$ and the above equation becomes

$$
c_{i \lambda}^{j}\left[-e_{j}\left(b_{k}^{i} \xi^{k}\right)+b_{t}^{k} b_{k j}^{i} \xi^{t}\right]=0, \quad \lambda=1, \ldots, N .
$$


(1.1) is a system of linear PDE of first order for $\xi=\left(\xi^{1}, \ldots, \xi^{n}\right)$ with $C^{\infty}$ coefficients. By a prolongation of (1.1) we shall mean a system of linear PDE obtained from (1.1) through a process of repeated differentiations, additions and multiplications by $C^{\infty}$ functions. The main result of this paper is the following.

Theorem 1.1. Let $G$ be a Lie subgroup of $G l(n ; \mathbf{R})$. Suppose that $\mathscr{O}$ and $\tilde{\mathscr{O}}$ are open neighborhoods of the origin of $\mathbf{R}^{n}$ with G-structure $P$ and $\widetilde{P}$, respectively. Let $f: \mathscr{O} \rightarrow \tilde{\mathscr{O}}$ be a G-mapping of class $C^{k}$ for some sufficiently large $k$. Suppose that the equation (1.1) for the infinitesimal automorphisms of $P$ has a prolongation to a complete system of order $m$ and that $\widetilde{P}$ has the same property. Then $f$ satisfies a complete system of order $m+1$.

Note that the existence of an infinitesimal automorphism on $\mathscr{O}$ or $\tilde{\mathscr{O}}$ is not assumed. In general, if a system of functions $f=\left(f^{1}, \ldots, f^{l}\right)$ satisfies a complete system of order $m$, then by introducing new variables for the partial derivatives of order $<m$, we can construct a Pfaffian system on a submanifold of $\mathbf{R}^{N}$ where $N=n+$ number of the newly introduced variables, so that $f$ may be identified with an integral manifold as in the proof of Theorem 1.1. So the questions of existence, regularity and uniqueness of $f$ reduce to the Frobenius theorem. We will call $G$-structures as in Theorem 1.1 Frobenius type.

Definition 1.2. $A$ G-structure $P$ on a $C^{\infty}$ manifold $M$ is a Frobenius type of order $m$ if the equation (1.1) for the infinitesimal automorphisms of $P$ in terms of any local coordinates has a prolongation to a complete system of order $m$.

We have

Corollary 1.3. Let $M$ and $\widetilde{M}$ be a $C^{\infty}$ manifolds with $G$-structure $P$ and $\widetilde{P}$, respectively, and $f: M \rightarrow \widetilde{M}$ be a G-mapping. If $P$ and $\widetilde{P}$ are Frobenius type of order $m$ and $f \in C^{m+1}$ then $f \in C^{\infty}$ and $f$ is locally determined by $\left\{D^{\beta} f(0):|\beta| \leq m\right\}$ at any point $0 \in M$.

Now let $P$ be a $G$-structure of Frobenius type of order $m$ on an open set $\mathscr{O} \subseteq \mathbf{R}^{n}$. Let

$$
D^{\alpha} \xi^{i}=H_{\alpha}^{i}\left(x, D^{\beta} \xi:|\beta|<m\right), \quad \forall \alpha \text { with }|\alpha|=m, \forall i=1, \ldots, n,
$$

be a prolongation of (1.1) to a complete system. Note that each $H_{\alpha}^{i}$ is linear in $D^{\beta} \xi$. To realize the process of prolongation in the jet spaces, we introduce new variables

$$
p_{\beta}=\left(p_{\beta}^{1}, \ldots, p_{\beta}^{n}\right) \text { for } D^{\beta} \xi=\left(D^{\beta} \xi^{1}, \ldots, D^{\beta} \xi^{n}\right) .
$$

For each positive integer $k$, the $k$ th order jet space is $J^{k} \equiv \mathscr{O} \times \mathbf{R}^{(k)}=$ $\{(x, \xi, p)\}$, where $p=\left(p_{\beta}:|\beta| \leq k\right)$ and $(k)$ is the number of the variables $(\xi, p)$. In $J^{k}$ consider the submanifold $\Delta^{k}$ which is defined by (1.1) and all 
the equations obtained by differentiating (1.1) in all possible ways up to order $k-1$. Let $X=\xi^{i}\left(\partial / \partial x_{i}\right)$ be a $C^{k}$ vector field. Then the $k$-graph of $X$ is the submanifold of $J^{k}$

$$
j_{X}^{k}(x) \equiv\left(x, \xi(x), D^{\beta} \xi(x):|\beta| \leq k\right)
$$

and $k$ th order contact systems is

$$
\Omega^{k} \equiv\left\{w \in T^{*} J^{k}:\left(j_{X}^{k}\right)^{*} w=0 \text {, for all vector fields } X \text { on } \mathscr{O}\right\} .
$$

Then $\Omega^{k}$ is spanned by

$$
\begin{aligned}
w^{k} & \equiv d \xi^{k}-p_{j}^{i} d x^{j} \text { and } \\
w_{\beta}^{i} & \equiv d p_{\beta}^{i}-p_{(\beta, j)}^{i} d x^{j}, \text { where }
\end{aligned}
$$

$(\beta, j)$ denotes the multi-index $\left(\beta_{1}, \ldots, \beta_{j}+1, \ldots, \beta_{n}\right)$ if $\beta=\left(\beta_{1}, \ldots, \beta_{n}\right)$, (cf. [8 and 9]). We prove

Lemma 1.4. Let $P$ be a $G$-structures on $\mathscr{O}$ and let $J^{k}, \Delta^{k}$ and $\Omega^{k}$ be as above. Then the following are equivalent:

(i) $P$ is of Frobenius type of order $m$;

(ii) the $(m-1)$ th contact system $\Omega^{m-1}$ defines an $n$-dimensional distribution $\mathscr{D}$ on $\Delta^{m-1}$, with $d x^{1} \wedge \cdots \wedge d x^{n} \neq 0$ on each integral element of $\mathscr{D}$.

Proof of Lemma 1.4. (i) $\Rightarrow$ (ii): Let (1.2) be a prolongation of (1.1) to a complete system. (1.2) is equivalent to the total differential equation

$$
d\left(D^{\beta} \xi^{i}\right)=H_{(\beta, j)}^{i}\left(x, D^{\gamma} \xi:|\gamma| \leq m-1\right) d x^{j}, \quad \forall \beta \text { with }|\beta|=m-1,
$$

$\forall i=1, \ldots, n$. This implies that on $\Delta^{m-1}$

$$
\Omega_{\beta}^{i} \equiv d p_{\beta}^{i}-H_{(\beta, j)}^{i}\left(x, \xi, p_{\gamma}\right) d x^{j}=0,
$$

$\forall \beta$ with $|\beta|=m=1, \forall i=1, \ldots, n$. Let $\mathscr{D}$ be the distribution on $\Delta^{m-1}$ given by $\Omega^{(m-1)}=0$. Then on each integral element of $\mathscr{D}$ we have

$$
\begin{aligned}
& d \xi^{i}=p_{j}^{i} d x^{j}, \\
& d p_{\beta}^{i}=p_{(\beta, j)}^{i} d x^{j}, \quad \forall \beta \text { with }|\beta|<m-1, \\
& d p_{\beta}^{i}=H_{(\beta, j)}^{i}(x, \xi, p) d x^{j}, \quad \forall \beta \text { with }|\beta|=m-1, \text { and } \\
& d x^{1} \wedge \cdots \wedge d x^{n} \neq 0 .
\end{aligned}
$$

Therefore, $\mathscr{D}$ is an $n$-dimensional distribution.

(ii) $\Rightarrow$ (i): Let $\mathscr{D}$ be the distribution as in (ii). Let $\varphi^{1}, \ldots, \varphi^{\nu}$ be differential 1-forms on $\Delta^{m-1}$ which generate the differential ideal associated with $\mathscr{D}$, where $\nu=\left(\right.$ dimension of $\left.\Delta^{m-1}\right)-n$. Set

$$
\varphi^{j}=a^{j} d x+b^{j} d \xi+c^{j} d p, \quad j=1, \ldots, \nu,
$$


where $a^{j}, b^{j}$ and $c^{j}$ are row vectors and $d x, d \xi$ and $d p$ are column vectors so that $a^{j} d x=a_{1}^{j} d x^{1}+\cdots+a_{n}^{j} d x^{n}$, etc. Since each integral element of $\mathscr{D}$ is $n$-dimensional subspace of $T\left(\Delta^{m-1}\right)$ on which $d x^{1} \wedge \cdots \wedge d x^{n} \neq 0$, we can solve $\varphi^{j}=0, j=1, \ldots, \nu$, for $d \xi$ and $d p$ we get

$$
\left\{\begin{aligned}
d \xi^{i} & =h_{j}^{i} d x^{j} \\
d p_{\beta}^{i} & =h_{(\beta, j)}^{i} d x^{j}, \quad \forall \beta \text { with }|\beta| \leq m-1, \quad \forall i=1, \ldots, n,
\end{aligned}\right.
$$

where $h$ are $C^{\infty}$ functions on $\Delta^{m-1}$.

This implies that if $\xi=\left(\xi^{1}, \ldots, \xi^{n}\right)$ satisfies (1.1) then

$$
d\left(D^{\beta} \xi^{i}\right)-h_{(\beta, j)}^{i}\left(x, D^{\gamma} \xi:|\gamma| \leq m-1\right) d x^{j}=0,
$$

for all $\beta$ with $|\beta| \leq m-1$. In particular, if $|\beta|=m-1$, the above equation is equivalent to

$$
D^{(\beta, j)} \xi^{i}=h_{(\beta, j)}^{i}\left(x, D^{\gamma} \xi:|\gamma| \leq m-1\right), \quad|\beta|=m-1,
$$

which is a complete system of order $m$. Q.E.D.

Proof of Theorem 1.1. Let (1.2) be the complete system for the infinitesimal automorphism of $P$ and let $\Omega^{k}$ be the $k$ th contact system on $\Delta^{k} \subseteq J^{k}$ for each $k=1, \ldots, m-1$. For each multi-index $\beta$ with $|\beta|=m-1$ and each $i=1, \ldots, n$, let $\Omega_{\beta}^{i} \equiv d p_{\beta}^{i}-H_{(\beta, j)}^{i} d x^{j}$, where $H$ are the same as in the complete system (1.2). Let $\mathscr{D}$ be the distribution as in Lemma 1.4. We will put tilde on the corresponding notions on $\widetilde{\mathscr{O}}: \widetilde{J}^{m-1}=\tilde{\mathscr{O}} \times \mathbf{R}^{(m-1)}=(\tilde{x}, \tilde{\xi}, \tilde{p})$, etc. A $C^{m+1}$ diffeomorphism $f: \mathscr{O} \rightarrow \tilde{\mathscr{O}}$ naturally defines a $C^{1}$ diffeomorphism $F^{k}: J^{k} \rightarrow \widetilde{J}^{k}$ for each $k=1, \ldots, m-1$ as follows: Let $F^{k}(x, \xi, p)=$ $(\tilde{x}, \tilde{\xi}, \tilde{p})$.

Then

$$
\begin{gathered}
\tilde{x}^{i}(x, \xi, p)=f^{i}(x) \\
\widetilde{\xi}^{i}(x, \xi, p)=\xi^{\lambda} \frac{\partial f^{i}}{\partial x^{\lambda}}, \quad i=1, \ldots, n
\end{gathered}
$$


and define $\widetilde{P}(x, \xi, p)$ by chain rule, namely

$$
\begin{aligned}
\tilde{p}_{j}^{i}(x, \xi, p) & =\frac{\partial \widetilde{\xi}^{i}}{\partial \tilde{x}^{k}} \\
& =\frac{\partial \widetilde{\xi}^{i}}{\partial x^{\mu}} \frac{\partial x^{\mu}}{\partial \tilde{x}^{j}}
\end{aligned}
$$

substitute (1.4) for $\xi^{i}$ and $p_{\mu}^{\lambda}$ for $\frac{\partial \xi^{\lambda}}{\partial x^{\mu}}$

$$
=\left(p_{\mu}^{\lambda} \frac{\partial f^{i}}{\partial x^{\lambda}}+\xi^{\lambda} \frac{\partial^{2} f^{i}}{\partial x^{\mu} \partial x^{\lambda}}\right) \frac{\partial x^{\mu}}{\partial \tilde{x}^{i}}
$$

each $\frac{\partial x^{\mu}}{\partial \tilde{x}^{j}}$ is an entry of $\left[\frac{\partial f^{i}}{\partial x^{j}}\right]_{i, j=1, \ldots, n}^{-1}$,

therefore a $C^{\infty}$ function of $\frac{\partial f^{i}}{\partial x^{j}}, i, j=1, \ldots, n$, so

$$
\begin{aligned}
= & \xi^{\lambda} \frac{\partial^{2} f^{i}}{\partial x^{\mu} \partial x^{\lambda}} \frac{\partial x^{\mu}}{\partial \tilde{x}^{j}}+a_{\lambda}^{\mu} p_{\mu}^{\lambda}, \\
& \text { where } a_{\lambda}^{\mu} \text { are } C^{\infty} \text { functions of }\left(D^{\gamma} f:|\gamma| \leq 1\right) .
\end{aligned}
$$

Now let $\beta=\left(\beta_{1}, \ldots, \beta_{n}\right)$ be a multi-index and $\left(j_{1}, \ldots, j_{|\beta|}\right)$ denotes the sequence $(\underbrace{1, \ldots, 1}_{\beta_{1} \text { times }}, \underbrace{2, \ldots, 2}_{\beta_{2} \text { times }}, \ldots, \underbrace{n, \ldots, n}_{\beta_{n} \text { times }})$.

Then by induction on $|\beta|$ we get

$$
\begin{aligned}
\tilde{p}_{\beta}^{i}(x, \xi, p)= & \xi^{\lambda}\left[\frac{\partial^{|\beta|+1} f^{i}}{\partial x^{\lambda} \partial x^{\lambda_{1}} \cdots \partial x^{\lambda_{|\beta|}}} \frac{\partial x^{\lambda_{1}}}{\partial \tilde{x}^{j_{1}}} \cdots \frac{\partial x^{\lambda_{1 \beta \mid}}}{\partial \tilde{x}^{j_{|\beta|}}}+a_{\beta, \lambda}\right] \\
& +a_{\beta, \lambda}^{\gamma} p_{\gamma}^{\lambda},|\gamma| \leq|\beta|,
\end{aligned}
$$

where $a$ are $C^{\infty}$ functions of $\left(D^{\gamma} f:|\gamma| \leq|\beta|\right)$. Then we claim

(1) $F^{m-1}\left(\Delta^{m-1}\right)=\widetilde{\Delta}^{m-1}$ and

(2) $F_{*}^{m-1}(\mathscr{D})=\widetilde{\mathscr{D}}$.

Proof of claim.

(1) A $C^{1}$ vector field $X=\xi^{i}\left(\partial / \partial x_{i}\right)$ is an infinitesimal automorphism of $P$ if and only if $f_{*} X$ is an infinitesimal automorphism of $\widetilde{P}$. This implies that $F^{1}\left(\Delta^{1}\right)=\tilde{\Delta}^{1}$. Then it is clear that $F^{k}\left(\Delta^{k}\right)=\tilde{\Delta}^{k}$ for $k=2, \ldots, m-1$.

(2) For each $k=1, \ldots, m-1$, we have $\left(F^{k}\right)^{*}\left(\tilde{\Omega}^{k}\right)=\Omega^{k}$ which is immediate from the definition of the contact system. In particular 


$$
\begin{aligned}
&\left(F^{m-1}\right)^{*}\left(\widetilde{\Omega}^{m-1}\right)=\Omega^{m-1} . \text { Thus we have } \\
& v \in \mathscr{D} \Leftrightarrow v \in T\left(\Delta^{m-1}\right) \text { and } v \text { annihilates } \Omega^{m-1} \\
& \Leftrightarrow F_{*} v \in T\left(\widetilde{\Delta}^{m-1}\right) \text { and } F_{*} v \text { annihilates } \widetilde{\Omega}^{m-1} \\
& \Leftrightarrow F_{*}^{m-1} v \in \widetilde{\mathscr{D}}, \quad \text { Q.E.D. }
\end{aligned}
$$

Now we compute $F^{*} \widetilde{\Omega}_{\beta}^{i},|\beta|=m-1$ :

$$
\begin{aligned}
(1.7)\left(F^{m-1}\right)^{*} \tilde{\Omega}_{\beta}^{i}= & \left(F^{m-1}\right)^{*}\left(d \tilde{p}^{i}-\widetilde{H}_{(\beta, j)}^{i}(\tilde{x}, \tilde{\xi}, \tilde{p}) d \tilde{x}^{j}\right) \\
& \text { substitute }(1.3)-(1.6) \text { for } \tilde{x}, \tilde{\xi} \text { and } \tilde{p}, \text { respectively, } \\
= & {\left[\frac{\partial^{m} f^{i}}{\partial x^{\lambda} \partial^{\lambda_{1}} \cdots \partial x^{\lambda_{m-1}}} \frac{\partial x^{\lambda_{1}}}{\partial \tilde{x}^{j_{1}}} \cdots \frac{\partial x^{\lambda_{m-1}}}{\partial \tilde{x}^{j_{m-1}}}+a_{\beta, \lambda}\right] d \xi^{\lambda} } \\
& +a_{\beta, \lambda}^{\gamma} d p_{\gamma}^{\lambda}, \quad|\gamma| \leq m-1 \\
& +\left[\xi^{\lambda} \frac{\partial^{m+1} f^{i}}{\partial x^{\lambda} \partial x^{\lambda_{1}} \cdots \partial x^{\lambda_{m-1}} \partial x^{k}} \frac{\partial x^{\lambda_{1}}}{\partial \tilde{x}^{j_{1}}} \cdots \frac{\partial x^{\lambda_{m-1}}}{\partial \tilde{x}^{j_{m-1}}}+b_{\beta, k}^{i}\right] d x^{k}
\end{aligned}
$$

where $a$ are $C^{\infty}$ functions of $\left(D^{\gamma} f:|\gamma| \leq m-1\right)$ and $b$ are $C^{\infty}$ functions of $\left(x, \xi, p, D^{\gamma} f:|\gamma| \leq m\right)$.

By the proof of Lemma 1.4, $\widetilde{D}$ on $\widetilde{\Delta}^{m-1}$ is given by

$$
\left\{\begin{aligned}
\widetilde{\Omega}^{m-1} & =0 \\
\widetilde{\Omega}_{\beta}^{i} & =0, \quad \forall i=1, \ldots, n, \quad \forall \beta \text { with }|\beta|=m-1 .
\end{aligned}\right.
$$

Recall that $\widetilde{\Omega}^{m-1}$ is the contact system and $\widetilde{\Omega}_{\beta}^{i}$ are the 1 forms defined by the complete system. Since $F_{*}^{m-1}(\mathscr{D})=\widetilde{D},\left(F^{m-1}\right)^{*} \widetilde{\Omega}_{\beta}^{i}$ is a linear combination of $\left\{\omega^{i}, \omega_{\gamma}^{i}, \Omega_{\delta}^{i}: i=1, \ldots, n,|\gamma|<m-1,|\delta|=m-1\right\}$, where $\omega$ are contact forms. So we set

$$
\begin{aligned}
\left(F^{m-1}\right)^{*} \widetilde{\Omega}_{\beta}^{i}= & c_{\beta, \lambda}^{i} \omega^{\lambda}+c_{\beta, \lambda}^{i, \gamma} \omega_{\gamma}^{\lambda}+c_{\beta, \lambda}^{i, \delta} \Omega_{\delta}^{\lambda} \\
= & c_{\beta, \lambda}^{i}\left(d \xi^{\lambda}-p_{k}^{\lambda} d x^{k}\right)+c_{\beta, \lambda}^{i, \gamma}\left(d p_{\gamma}^{\lambda}-p_{(\gamma, k)}^{\lambda} d x^{k}\right) \\
& +c_{\beta, \lambda}^{i, \delta}\left(d p_{\delta}^{\lambda}-H_{(\delta, k)}^{\lambda} d x^{k}\right),
\end{aligned}
$$

where $c$ are $C^{1}$ functions on $\Delta^{m-1},|\gamma| \leq m-2$ and $|\delta|=m-1$.

By equating the components of $d \xi$ and $d p$ in (1.7) and (1.8) we get $c$ 's as $C^{\infty}$ functions of $\left(x, \xi, p, D^{\gamma} f:|\gamma| \leq m\right)$ for $(x, \xi, p) \in \Delta^{m-1}$. Substitute this in (1.8) and equate the components of $d x^{k}$ in (1.7) and (1.8) to get

$$
\begin{aligned}
& \xi^{\lambda} \frac{\partial^{m+1} f^{i}}{\partial x^{\lambda} \partial x^{\lambda_{1}} \cdots \partial x^{\lambda_{m-1}} \partial x^{k}} \frac{\partial x^{\lambda_{1}}}{\partial \tilde{x}^{j_{1}}} \cdots \frac{\partial x^{\lambda_{m-1}}}{\partial \tilde{x}_{m-1}^{j}} \\
= & C^{\infty} \text { function of }\left(x, \xi, p, D^{\gamma} f:|\gamma| \leq m\right),
\end{aligned}
$$


where $(x, \xi, p) \in \Delta^{m-1}$. Since (1.1) is a system of linear partial differential equations of first order obtained from the structure equations of the Lie Algebra $\mathscr{G}$, we see that $d x^{1} \wedge \cdots \wedge d x^{n} \wedge d \xi^{1} \cdots d \xi^{n} \neq 0$ on $\Delta^{1} \subseteq J^{1}$ and therefore on $\Delta^{m-1} \subseteq J^{m-1}$. Thus there exists a $C^{\infty}$ function $p(x, \xi)$ such that $(x, \xi, p(x, \xi)) \in \Delta^{m-1}, \forall(x, \xi)$. For each $j=1$, the restriction of (1.9) to the submanifold $\{(x, \xi, p(x, \xi)): \xi=(0, \ldots, 0, \underbrace{1}_{j t h}, 0, \ldots, 0)\}$ is

$$
\begin{aligned}
& \frac{\partial^{m+1} f^{i}}{\partial x^{j} \partial x^{\lambda_{1}} \cdots \partial x^{\lambda_{m-1}} \partial x^{k}} \frac{\partial x^{\lambda_{1}}}{\partial \tilde{x}^{j_{1}}} \cdots \frac{\partial x^{\lambda_{m-1}}}{\partial \tilde{x}^{j_{m-1}}} \\
& =C^{\infty} \text { function of }\left(x, D^{\gamma} f:|\gamma| \leq m\right) .
\end{aligned}
$$

Here $i, j, j_{1}, \ldots, j_{m-1}$ and $k$ are arbitrary. Since the matrix

$$
\left(\partial x^{i} / \partial \tilde{x}^{j}\right)_{i, j=1, \ldots, n}
$$

is nonsingular and each $\partial x^{i} / \partial \tilde{x}^{j}$ is a $C^{\infty}$ function of $(D f)$, from (1.10) we get

$$
\frac{\partial^{m+1} f^{i}}{\partial x^{j} \partial x^{j_{1}} \cdots \partial x^{j_{m-1}} \partial x^{k}}=C^{\infty} \text { function of }\left(x, D^{\gamma} f:|\gamma| \leq m\right) .
$$

This completes the proof.

\section{2. $G$-STRUCTURES OF FINITE ORDER}

Let $G$ be a Lie subgroup of $G l(n ; \mathbf{R})$ and $\mathscr{G}$ be the associated Lie algebra. The $k$ th prolongation $\mathscr{G}^{(k)}$ of $\mathscr{G}$ is the space of symmetric multilinear mappings

$$
t: \underbrace{\mathbf{R}^{n} \times \cdots \times \mathbf{R}^{n}}_{(k+1) \text { times }} \rightarrow \mathbf{R}^{n}
$$

such that, for each fixed $v_{1}, \ldots, v_{k} \in \mathbf{R}^{n}$, the linear transformation

$$
v \in \mathbf{R}^{n} \mapsto t\left(v, v_{1}, \ldots, v_{k}\right) \in \mathbf{R}^{n} \text { belongs to } \mathscr{G} \text {. }
$$

$G$ is said to be of finite order $k$ if $\mathscr{G}^{(k)}=0$ and $\mathscr{G}^{(k-1)} \neq 0$. Riemannian structures and conformal structures (when dimension $\geq 3$ ) are of finite order 1 and 2 , respectively (cf. [5 or 6]).

Theorem 2.1. Let $M$ be a $C^{\infty}$ manifold of dimension $n$ and $P$ be $a \quad G$ structure on $M$. If $G$ is of finite order $m-1(m \geq 1)$, then $P$ is of Frobenius type of order $m$.

Proof. Since $\mathscr{G}$ is a linear subspace of $g l(n ; \mathbf{R})$, it is defined by $\mathscr{G}=\left\{\left(y_{j}^{i}\right) \in\right.$ $\left.g l(n ; \mathbf{R}): \sum_{i, j=1}^{n} c_{i \lambda}^{j} y_{j}^{i}=0, \lambda=1, \ldots, N\right\}$, where the $c_{i \lambda}^{j}$ are constants and $N$ is the codimension of $\mathscr{G}$ in $g l(n ; \mathbf{R})$. Therefore, as a linear space, $\mathscr{G}^{(m-1)}$ is isomorphic to the subspace of

$$
\mathbf{R}^{n^{m+1}}=\left(y_{j_{1} \cdots j_{m}}^{i}\right), \quad \text { each } i, j \in\{1, \ldots, n\},
$$


which is defined by the following system of linear equations:

$$
\sum_{i, j_{1}=1}^{n} c_{i \lambda}^{j_{1}} y_{j_{1} \cdots j_{m}}^{i}=0, \quad \lambda=1, \ldots, N
$$

and

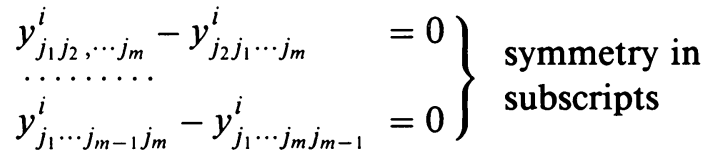

Since the only solution of $(2.11)$ is $y=0$, there exists $n^{m+1}$ independent equations in (2.11). Let

$$
g^{1}(y)=0, \ldots, g^{n^{m+1}}(y)=0 .
$$

Now let $M$ be a $C^{\infty}$ manifold of dimension $n$ with a $G$-structure $P$. We fix a frame $\left(e_{1}, \ldots, e_{n}\right)$ belonging to $P$. Let $X=\sum_{i=1}^{n} \xi^{i} e_{i}$ be an infinitesimal automorphism of $P$.

Define $\xi_{j}^{i}$ by $\left[e_{j}, X\right]=\sum_{i=1}^{n} \xi_{j}^{i} e_{i}$. Then the matrix $\left[\xi_{j}^{i}\right]$ belongs to $\mathscr{G}$. For any sequence $\left(j_{2}, \ldots, j_{k}\right)$, each $j \in\{1, \ldots, n\}$, we denote by $\xi_{j j_{2} \cdots j_{k}}^{i}$ the derivative of $e_{j_{k}} \cdots e_{j_{2}}\left(\xi_{j}^{i}\right)$. Then in the Jacobi identity

$$
\left[e_{k},\left[e_{j}, X\right]\right]-\left[e_{j},\left[e_{k}, X\right]\right]=\left[\left[e_{k}, e_{j}\right], X\right]
$$

substitute $\sum_{i=1}^{n} \xi_{j}^{i} e_{i}$ and $\sum_{i=1}^{n} \xi_{k}^{i} e_{i}$ for $\left[e_{j}, X\right]$ and $\left[e_{k}, X\right]$, respectively, we get $\xi_{j k}^{i}-\xi_{k j}^{i}=\left\langle\xi^{\lambda}, \xi_{\mu}^{\lambda}\right\rangle$, where $\langle$,$\rangle denotes a linear combination of the variables$ inside with $C^{\infty}$ coefficients. By induction on the number of the subscripts we see that a transposition for any two subscripts in $\xi_{j_{1} \cdots j_{k}}^{i}$ makes a difference by a linear combination of $\left\{\xi_{J}^{\lambda}:|J|<k, \lambda=1, \ldots, n\right\}$, where $J=\left(j_{1} j_{2} \cdots\right)$ is a sequence of subscripts and $|J|$ is the size of $J$. Moreover, since $\mathscr{G}$ is a linear space, for each fixed $j_{2} \cdots j_{k}$, the matrix of the derivatives

$$
\left[\xi_{j_{1} j_{2} \ldots j_{k}}^{i}\right]_{i, j_{1}=1, \ldots, n} \text { belongs to } \mathscr{G} \text {. }
$$

Now at each point $x \in M$, consider

$$
\sum_{i, j_{1}=1}^{n} c_{i \lambda}^{j_{1}} \xi_{j_{1} j_{2} \cdots j_{m}}^{i}=0, \lambda=1, \ldots, N
$$

and

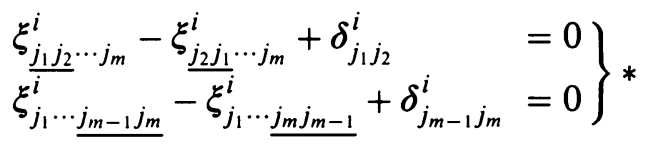

* is the symmetry in the subscripts modulo lower order terms $\delta$, where each $\delta$ is a linear combination of $\left\{\xi_{J}^{t}:|J| \leq m-1, t=1, \ldots, n\right\}$ with $C^{\infty}$ coefficients. Let

$$
g^{1}(x, \xi)=0, \ldots, g^{n^{m+1}}(x, \xi)=0,
$$


be the equations corresponding to (2.12). Since the last $n^{m+1}$ columns of the Jacobian matrix $(\partial g(x, \xi) / \partial \xi)$ is equal to $(\partial g / \partial y)$, which is nonsingular, we can solve (2.14) to get $\xi_{j_{1}, \ldots j_{m}}^{i}=$ a linear combination of $\left\{\xi_{J}^{t}:|J| \leq m-1\right.$, $t=1, \ldots, n\}$ with $C^{\infty}$ coefficients, for each $i, j_{1}, \ldots, j_{m}$. This completes the proof.

\section{REMARKS ON CR STRUCTURES}

A CR structure $P$ of complex dimension $n$ and CR codimension $d$ on a $C^{\infty}$ manifold $M$ if dimension $2 n+d$ is a $G$-structure, where $G$ is the group of all the matrices of the form

$$
\left[\begin{array}{ll}
A & * \\
0 & B
\end{array}\right] \text {, where } A \in G l(n ; \mathbf{C}) \subset G L(2 n ; \mathbf{R}) \text { and } B \in G l(d ; \mathbf{R}) \text {. }
$$

Let $\left(e_{1}, \ldots, e_{2 n+d}\right)$ be a frame field belonging to $P$. Let $H(M)$ be the sub-bundle of the tangent bundle $T(M)$ spanned by $\left(e_{1}, \ldots, e_{2 n}\right)$. We assume the integrability condition on $H(M)$, as usual (cf. [2]). This group $G$ is of infinite order, for the associated Lie algebra of $G$ contains a matrix or rank 1 (Proposition 1.4 of [5]). However, under certain conditions on the Levi form $P$ is of Frobenius type. When $d=1$ the following is well known: If $M$ and $\widetilde{M}$ are $C^{\infty}$ CR manifolds with nondegenerate Levi forms, and $f: M \rightarrow \widetilde{M}$ is a CR diffeomorphism then

(1) $f$ is locally determined by a finite number of constants, and

(2) $f \in C^{7}$ implies that $f \in C^{\infty}$.

These are consequences of the existence of the invariant Cartan connection on the bundle of pseudoconformal frames over $M$ (cf. [2,7]). From the viewpoint of this paper the above (1) and (2) follow from the fact that a nondegenerate CR structure of CR codimension 1 is of Frobenius type of order 3. This can be easily shown by the method used in [3] and [4], where direct construction of complete system of mappings have been treated.

When $M$ and $\widetilde{M}$ are real analytic hypersurfaces in $\mathbf{C}^{n}$, regularity of $\mathrm{CR}$ mappings has been studied in [1] and [7] in relation to the problem of holomorphic extension of CR mappings. For abstract $C R$ manifolds, the greater the $C R$ codimension $d$ is, the more conditions on $P$ are required in order for mappings to be regular. Our further problem is to find conditions for given $n$ and $d$, under which CR structure $P$ becomes Frobenius type, or more generally, to find conditions on a nonelliptic $G$-structure (cf. [5]) which imply the regularity of transformations.

\section{ACKNOWLEDGMENTS}

The author thanks D. Burns for discussions and his encouragement and S. Webster for helpful conversations.

The author is grateful to the referee who pointed out mistakes of the first draft and gave helpful suggestions. 


\section{REFERENCES}

1. M. S. Baouendi, H. Jacobowitz and F. Treves, On the analyticity of CR mappings, Ann. of Math. (2) 122 (1985), 365-400.

2. S. S. Chern and J. K. Moser, Real hypersurfaces in complex manifolds, Acta Math. 133 (1974), 219-271.

3. C. Han, Analyticity of $C R$ equivalences between some real hypersurfaces in $\mathbf{C}^{n}$ with degenerate Levi forms, Invent. Math. 73 (1983), 51-69.

4. __ Regularity and uniqueness of certain systems of functions annihilated by a formally integrable system of vector fields, Rocky Mountain J. Math. (to appear).

5. S. Kobayashi, Transformation groups in differential geometry, Chapter 1, Springer-Verlag, Berlin and New York, 1972.

6. S. Sternberg, Lectures on differential geometry, Chapter 7, Prentice-Hall, Englewood Cliffs, N. J., 1964.

7. S. Webster, Analytic discs and the regularity of $C R$ mappings of real submanifolds in $\mathbf{C}^{n}$, Proc. Sympos. Pure Math., vol. 41, Amer. Math. Soc., Providence, R. I., 1984, pp. 69-79.

8. R. Gardner, Differential geometric methods interfacing control theory, Differential Geometric Control Theory (R. Brocket, ed.), Birkhauser, Boston, Mass., 1983, pp. 117-180.

9. P. Olver, Applications of Lie groups to differential equations, Chapter 2, Springer-Verlag, Berlin and New York, 1986.

Department of Mathematics, Texas Tech University, Lubbock, Texas 79409

Current address: Department of Mathematics, Pohang Institute of Science and Technology, Pohang 790, South Korea 